\section{NLRP3, \\ un inflammasome sous contrôle}

\author{
Marine Groslambert ${ }^{1-5}$, Bénédicte F. Py ${ }^{1-5, *}$
}

> La réponse immunitaire innée protège l'organisme par la détection rapide des agents pathogènes et des lésions via des récepteurs spécialisés, dont NLRP3. Celui-ci assemble un inflammasome, un complexe cytosolique de signalisation qui active la caspase-1, contrôle la libération de cytokines et de facteurs inflammatoires produits dans le cytosol comme les interleukines $1 \alpha / \beta$. Les pathologies inflammatoires associées à NLRP3, dont la goutte, révèlent la nécessité d'un contrôle étroit de son activité. Cette revue présente les avancées sur la signalisation du priming (ou amorçage) du complexe puis de son activation avec une attention particulière sur le rôle des modifications post-traductionnelles de NLRP3. <

Les inflammasomes sont des plateformes de signalisation multiprotéiques assemblées par des récepteurs cytosoliques qui détectent les agents pathogènes, $(\rightarrow)$ Voir la Synthèse de Y. Jamilloux et T. Henry, $m / s n^{\circ} 11$, novembre 2013, page 975 ou les dommages subis par la cellule [1] $(\rightarrow)$. Leur formation aboutit à l'activation des caspases inflammatoires (les caspases- 1 et 11 chez la souris, les caspases-1, 4 et 5 chez l'homme). Ces protéases sont à l'origine de la pyroptose, une forme de mort cellulaire pro-inflammatoire, en induisant le clivage d'une protéine cytosolique, appelée Gasdermine-D [2, 3] $\rightarrow$ ) dont le domaine $\mathrm{N}$-terminal,

$(\rightarrow)$ Voir la Synthèse de L. Cabon et al., $\mathrm{m} / \mathrm{s}$ $n^{\circ} 12$, décembre 2013, page 1117 alors libéré, s'oligomérise pour former des pores cytolytiques qui s'insèrent dans la membrane plasmique. La caspase-1 contrôle également la maturation, par un clivage direct, de pro-cytokines cytosoliques, dont I'IL(interleukine)-1 $\beta$ et l'IL-18. Un mécanisme de sécrétion non conventionnelle contrôlé par la caspase-1, et surtout la rupture de la membrane plasmique due à la pyroptose, permettent ensuite la libération de ces

Vignette (Photo @ Inserm - Elisabeth Génot).

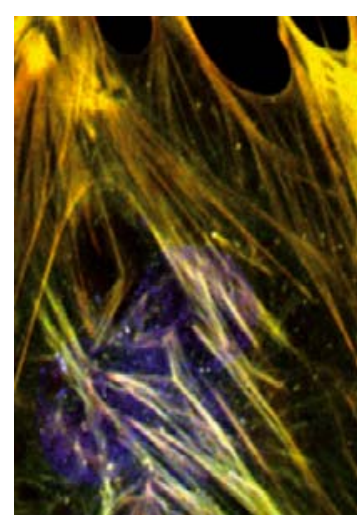

${ }^{1} \mathrm{CIRI}$, Centre international de recherche en infectiologie, université de Lyon, 21, avenue Tony Garnier, 69007 Lyon, France. ${ }^{2}$ Inserm, U1111, Lyon, France. ${ }^{3}$ École normale supérieure de Lyon, 69000 Lyon, France. ${ }^{4}$ Université Lyon 1, Centre international de recherche en infectiologie, 69000 Lyon, France.

${ }^{5}$ CNRS, UMR5308, Lyon, France. benedicte.py@inserm.fr

cytokines et d'autres facteurs intracellulaires pro-inflammatoires dont I'IL-l $\alpha$. La mort par pyroptose de cellules infectées permet d'éliminer en parallèle les niches de réplication des pathogènes intracellulaires. La structure des inflammasomes dits non-canoniques, associés à la caspase-11, ou 4 et 5 chez l'homme, reste mal connue. Les inflammasomes canoniques, qui sont eux, associés à la caspase-1, sont assemblés par des récepteurs cytosoliques de la famille des NLR (nucleotide-binding domain leucin-rich repeat) ou ALR (Aim2-like receptors). Ces récepteurs s'oligomérisent pour former une plateforme de nucléation pour des filaments formés par la protéine adaptatrice ASC (apoptosis-associated speck-like protein containing a CARD domain, aussi appelé PyCARD, CARD5, TMS1, Q9ULZ3), dont la surface permet, à son tour, la nucléation de filaments de pro-caspase-1 [4]. Ces inflammasomes forment des specks (ou structures ponctiformes) d'une taille de l'ordre du micron, visibles en microscopie, et qui sont uniques pour une cellule.

NLRP3 (nucleotide-binding domain leucin-rich repeat [LRR] and pyrin-containing receptor 3 , nommé également cryopyrin, CIASI [cold autoinflammatory syndrome 1 protein] ou NALP3 [NACHT, LRR and PYD domains-containing protein 3]) a été initialement décrit pour son association génétique avec des syndromes auto-inflammatoires héréditaires monogéniques dominants, nommés CAPS (cryopyrin-associated periodic syndrome) ou cryopyrinopathies [5]. Depuis, NLRP3 a été impliqué dans de nombreuses pathologies multifactorielles à composante inflammatoire, dont l'arthrite goutteuse, l'athérosclérose, le diabète de type 2 et la stéatose hépatique non-alcoolique $[6,7]$. II est également impliqué dans plusieurs maladies neurodégénératives, dont la sclérose en plaques et les maladies d'Alzheimer et de Parkinson, ainsi que dans les pathologies associées au vieillissement [6-9]. NLRP3 est aussi important au cours des infections par différents pathogènes. II intervient en effet dans la reconnaissance de 
divers pathogènes comme certaines amibes, des protozoaires, dont Plasmodium, des champignons, comme Candida albicans ou Saccharomyces cerevisiae, des bactéries parmi lesquelles Staphylococcus aureus et Listeria monocytogenes, et de divers virus tels que le virus de la grippe (influenza), le virus Sendaï, et des adénovirus [10]. L'impact de NLRP3 dans la réponse à ces pathogènes reste partiel, en raison de la redondance des récepteurs de l'immunité innée.

NLRP3 est une protéine de 115 kDa constituée (1) d'un domaine LRR (leucin-rich repeat) C-terminal, dont la fonction régulatrice est mal comprise, (2) d'un domaine central d'oligomérisation (NOD, nucleotide-binding and oligomerization domain ou NACHT) qui présente une activité ATPase, et (3) d'un domaine N-terminal effecteur PyD (pyrin domain) capable de recruter l'adaptateur ASC. De nombreux motifs moléculaires (les PAMP ou les DAMP, pour pathogen ou damage-associated molecular pattern) qui sont associés aux pathogènes ou aux réponses pathologiques de la cellule, activent NLRP3. On peut citer, par exemple, l'hyperglycémie, les céramides, certains acides gras, des agrégats protéiques (amyloïde- $\beta$, IAPP [islet amyloid polypeptide]), des cristaux (de cholestérol, d'urate de sodium, d'amiante [11] $(\rightarrow)$, de silice, de pyrophosphate $(\rightarrow)$ Voir la Nouvelle de C. Dostert et V. Pétrilli, $m / s n^{\circ} 11$, novembre 2013, page 975 de calcium, d'alun), I'ATP extracellulaire, l'acide hyaluronique, les toxines bactériennes formant des pores, certains acides nucléiques bactériens et viraux, ou encore l'hémozoïne (pigment malarique) produit lors de l'infection par Plasmodium [12]. Comme le suggère la grande diversité structurale de ces activateurs, NLRP3 n'est donc pas un récepteur stricto sensu qui lie des PAMP ou des DAMP. II s'agit plus d'un senseur de stress cytosolique. Dans cette revue, nous présentons les avancées récentes de la compréhension des voies de signalisation qui contrôlent l'activation de NLRP3. Une attention particulière est portée au rôle clé des modifications post-traductionnelles de NLRP3 pour l'assemblage de l'inflammasome.

\section{Les voies de signalisation qui contrôlent l'assemblage de l'inflammasome NLRP3}

Dans les macrophages, l'assemblage de l'inflammasome NLRP3 nécessite deux signaux, dits de priming (ou amorçage, signal 1) et d'activation (signal 2) (Figure 1). Les signaux de priming peuvent être des cytokines ou des PAMP/DAMP qui sont reconnus par des récepteurs qui initient la transcription de nombreux gènes. Les signaux d'activation correspondent, quant à eux, à différents stress cellulaires. Des conditions identiques semblent exister pour l'assemblage de l'inflammasome NLRP3 dans d'autres types cellulaires, moins étudiés que les macrophages, dont les monocytes, les mastocytes ou les neutrophiles. Néanmoins, dans les monocytes humains, le signal de priming peut suffire à l'assemblage de l'inflammasome, dans certaines conditions $[13,14]$.

Le priming de NLRP3 se produit à la suite de l'activation par leurs ligands de récepteurs, dont les TLR (Toll-like receptors), NOD2 (nucleotidebinding oligomerization domain 2), ou RAGE (receptor for advanced glycation end products), pour les PAMP/DAMP, ou, pour les cytokines, les récepteurs du TNF (tumor necrosis factor), des $\mathrm{IL}-1$, et les récepteurs des anaphylatoxines $\mathrm{C} 3 \mathrm{a}$ et $\mathrm{C} 5 \mathrm{a}$ du système du complément $[15,16]$. Une production excessive d'espèces réactives de l'oxygène (ou ROS, reactive oxygen species) permet également le priming de NLRP3. Plusieurs voies de priming sont successivement activées lors de l'engagement des TLR. La première à avoir été décrite est celle qui implique l'activation de la transcription induite par ces récepteurs et la synthèse protéique de novo qui en résulte [15]. Ce priming, dit transcriptionnel, est effectif plusieurs heures après l'engagement des récepteurs. II dépend de MyD88 (myeloid differentiation primary response 88), TRIF (TIR domaincontaining adapter-inducing interferon- $\beta$ ), FADD (Fas associated protein with death domain) et caspase-8, ainsi que des ROS, qui activent le facteur NF- $\kappa B$ (nuclear factor-kappa B) $[17,18]$. L'expression de NLRP3, alors fortement augmentée, suffit à se substituer au priming. II a donc été suggéré que le niveau basal de NLRP3 serait insuffisant pour l'assemblage de l'inflammasome et que le priming reposerait alors sur l'augmentation de son expression. Ce priming transcriptionnel est nécessaire pour l'expression de certains substrats de l'inflammasome, dont la pro-IL-1 $\beta$. Par contre, les expressions d'ASC, de pro-caspase- 1 et d'autres substrats de NLRP3, comme la pro-IL-18, sont constitutives et ne sont en revanche pas affectées par ce priming.

À côté du priming transcriptionnel, l'engagement des TLR et du récepteur des IL-1 (IL-IR), mais pas des récepteurs du TNF (TNFR), permet très précocement et transitoirement, un priming de NLRP3, indépendamment de la transcription et de la synthèse protéique [19]. Le niveau basal de NLRP3 suffirait donc à l'assemblage de l'inflammasome, ce qui suggère que le priming transcriptionnel repose sur l'expression d'autres facteurs qui restent à définir. Les mécanismes de priming sont donc multiples. À des étapes précoces, ils reposent uniquement sur des modifications post-traductionnelles. Ce priming non-transcriptionnel est opérationnel très rapidement (dès 10 à 30 min après l'engagement des TLR, par exemple) via la voie MyD88/IRAKI/4 (IL-I receptor-associated kinase $1 / 4$ ), après activation de TLR4 et de TLR2, et/ou légèrement plus tardivement (entre $30 \mathrm{~min}$ et $\mathrm{l} \mathrm{h}$ ), par la voie de signalisation TRIF/ IRAKl/FADD/caspase-8/RIPl (receptor-interacting protein 1), initiée par l'activation de TLR4 et de TLR3. Il dépend également des ROS mitochondriales qui suffisent à se substituer à l'engagement des TLR [19-21]. Dans les phases tardives (entre 12 et $24 \mathrm{~h}$ ), l'engagement de TLR4, mais pas des TNFR ou de TLR2, produit une désensibilisation de NLRP3 vis-à-vis des signaux d'activation. Deux mécanismes de désensibilisation, 


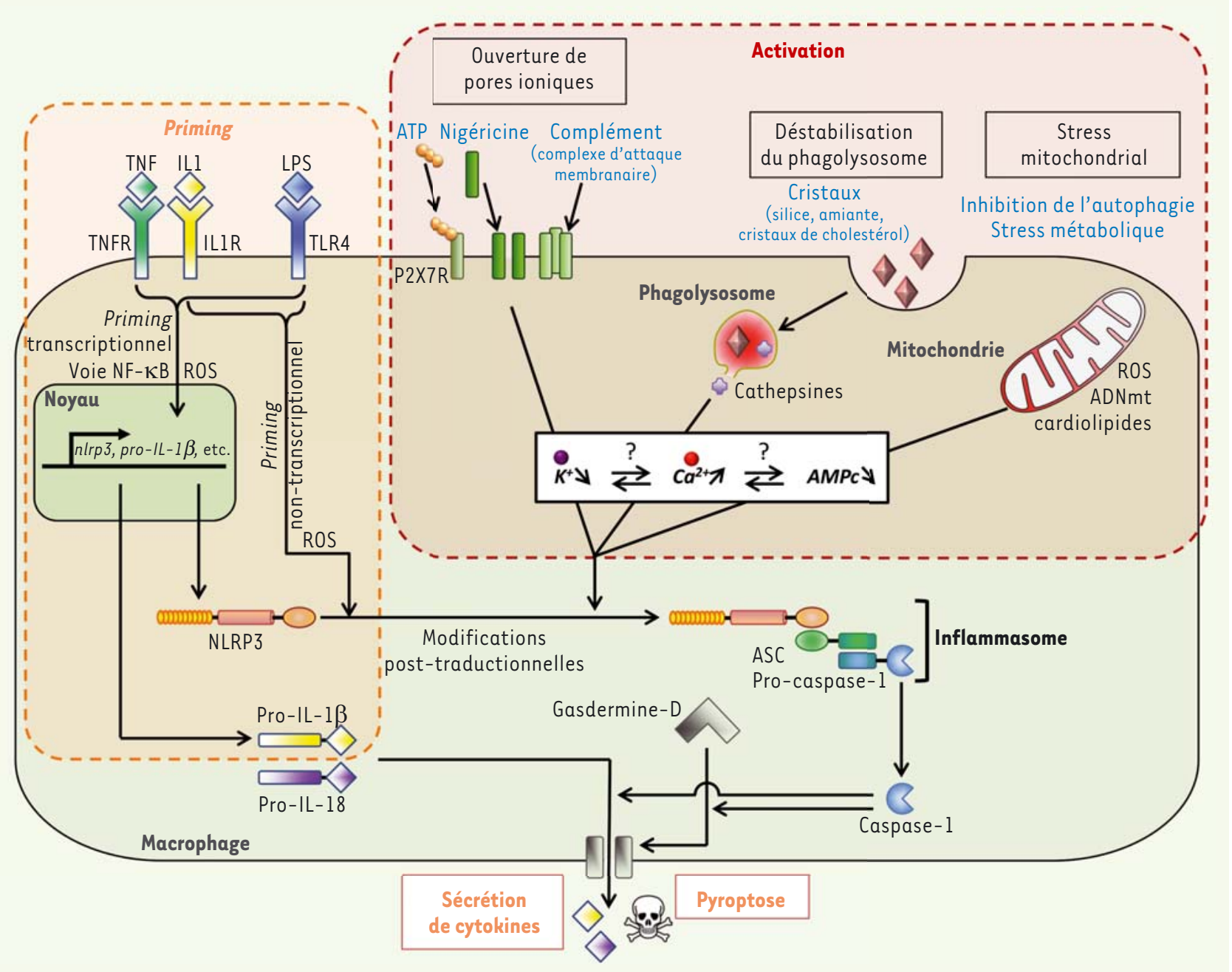

Figure 1. Voies de signalisation contrôlant l'assemblage de l'inflammasome. Le priming de NLRP3 (nucleotide-binding, LRR and PYD domainscontaining protein 3) dépend de l'activation d'autres récepteurs de l'immunité innée ou de récepteurs de cytokines. Son activation est le résultat de différents stress cellulaires ciblant, entre autres, la perméabilité ionique de la membrane plasmique, les lysosomes ou les fonctions mitochondriales, qui perturbent l'homéostasie ionique du cytoplasme. Reçus de façon coordonnée, ces deux signaux conduisent à des modifications post-traductionnelles de NLRP3 permettant l'assemblage de l'inflammasome, l'activation de la caspase-1, le clivage de la Gasdermine-D et la pyroptose, ainsi que la maturation et la sécrétion de cytokines. TNF : tumor necrosis factor; TNFR : tumor necrosis factor receptor ; ILl : interleukin-1 ; ILIR : interleukin-1 receptor; IL-18 : interleukin-18; LPS : lipopolysaccharide; TLR : Toll-like receptor; NF- $\mathrm{KB}$ : nuclear factor-kappa B ; ROS : reactive oxygen species; ATP : adenosine triphosphate; P2X7R : purinergic receptor P2X 7 ; AMPc : cyclic adenosine monophosphate; ASC: apoptosis-associated speck-like protein containing a CARD domain.

au moins, ont été documentés: l'induction de la protéine p62 (ou sequestosome 1) et la dégradation de NLRP3 par autophagie, et/ou la sécrétion d'IFN-I (interféron de type I, IFN $\alpha / \beta$ ) qui initie la production d'oxyde nitrique (NO) et inhibe NLRP3 [16, 22]. La combinaison de différentes voies de signalisation, en aval de TLR4, permet ainsi successivement, un priming non-transcriptionnel, puis transcriptionnel et, finalement, une désensibilisation de NLRP3. Bien que peu étudié, ce déroulé semble être différent lors de l'engagement d'autres récepteurs de priming, comme les TNFR. L'impact respectif de ces différentes voies in vivo en contexte infectieux ou auto-inflammatoire, reste cependant encore mal compris.
À la suite du priming, NLRP3 devient compétent pour être activé. Différents signaux d'activation, classés selon le type de stress cellulaire qu'ils induisent, ont été décrits. Les premiers provoquent l'ouverture de pores dans la membrane plasmique. C'est le cas de certaines toxines bactériennes, dont la nigéricine (produite par Streptomyces hygroscopicus), la forme clivée de la Gasdermine-D, qui contrôle l'activation de NLRP3 en aval de la caspase-11, ou le complexe d'attaque membranaire du complément [23]. L’ATP, libéré dans le milieu par les cellules nécrosées, permet également 
l'ouverture de pores via le récepteur P2X7 (purinergic receptor P2X 7) [24]. La deuxième catégorie de signaux correspond à des structures particulaires comme l'amiante, la silice, les cristaux de cholestérol, l'amyloïde- $\beta$ et les liposomes qui, en s'accumulant dans les lysosomes après avoir été phagocytées, entraînent leur rupture et la libération de cathepsines et de $\mathrm{Ca}^{2+}$ dans le cytosol [25]. Les specks, qui contiennent l'inflammasome, sont également libérés dans le milieu extracellulaire par les cellules pyroptotiques; ils sont ensuite phagocytés par les macrophages, leur ingestion provoquant l'activation de l'inflammasome, par la déstabilisation des lysosomes, et celle de la caspase-1, par un recrutement direct, ce qui amplifie la réponse des cellules [26, 27]. Les perturbateurs des fonctions mitochondriales forment une troisième catégorie de signaux. Ils comprennent les stress métaboliques et les inhibiteurs de la chaîne respiratoire mitochondriale ou de la mitophagie ${ }^{1}$ [28]. De nombreux autres signaux activateurs de NLRP3 n'entrent dans aucune de ces catégories, comme les rayons ultra-violets ou l'activation par le calcium extracellulaire de récepteurs couplés aux protéines $G$ [29]. Ces stimulus sont à l'origine de différentes signalisations qui activent NLRP3 [12]. Bien qu'aucun élément de convergence absolu ne permette, encore, d'établir un consensus, certaines voies apparaissent communes à de nombreux activateurs comme (1) la mise en place d'un efflux de potassium, qui produit un changement conformationnel des oligomères NLRP3; (2) une augmentation du calcium cytosolique, via l'ouverture des canaux ioniques TRP (transient receptor potential) ou la signalisation PLC/ IP3R (phospholipase C/inositol 3 phosphate receptor), qui favoriserait directement le recrutement d'ASC par NLRP3 et/ou qui diminuerait la production d'AMPc, inhibiteur de l'inflammasome; (3) les dommages mitochondriaux - qui peuvent être consécutifs à l'entrée du calcium dans les mitochondries - conduisant à la production de ROS, à la libération d'ADN mitochondrial et à l'exposition de cardiolipides à la membrane externe des mitochondries [29, 30]. Ces deux derniers ont été proposés comme des activateurs interagissant directement avec NLRP3 [31, 32].

L'assemblage de l'inflammasome NLRP3 est donc finement contrôlé par la coordination des étapes de priming et d'activation. Les voies de signalisation et les mécanismes moléculaires impliqués sont encore mal caractérisés. Toutefois, plusieurs travaux récents révèlent l'importance des modifications post-traductionnelles de NLRP3 dans ce processus (Figure 2).

\section{Modifications post-traductionnelles de l'inflammasome NLRP3 et contrôle de son assemblage}

Le priming de NLRP3 est donc en partie indépendant de la transcription. Très récemment, un rôle clé a été révélé pour la phosphorylation de la sérine S198 (sérine en position 198) de NLRP3 chez l'homme (qui correspond à la sérine S194 chez la souris), par JNKl (c-Jun N-terminal kinase 1). La phosphorylation de cette sérine est un événement commun au priming transcriptionnel et non-transcriptionnel. Elle a

${ }^{1}$ Une forme d'autophagie s'adressant spécifiquement aux mitochondries. pour origine les voies de signalisation impliquant MyD88 et/ou TRIF, et sa cinétique peut être reliée au priming de NLRP3 par ces voies [33]. Cette phosphorylation induit une déubiquitination de NLRP3, et semble favoriser son oligomérisation. Un polymorphisme de NLRP3 interférant avec le processus de phosphorylation de la sérine (S198N) a été identifié chez un patient présentant un CAPS. Néanmoins, ce patient était également porteur d'une autre mutation, bien caractérisée dans ce syndrome (y570C), et sans doute responsable de la pathologie [34]. Ces résultats sont cohérents avec l'observation plus ancienne d'une déubiquitination de NLRP3 dépendant des ROS mitochondriales produites lors du priming non-transcriptionnel induit par un bref engagement de TLR4 [20]. Cependant aucune $\varepsilon 3$ ubiquitine ligase ou déubiquitinase, impliquée dans ce priming court, n'a été identifiée. Les chaperonnes SGTl/ HSP9O (suppressor of the G2 allele of skpl/heat shock protein 90), qui sont associées, à l'état basal, à NLRP3 et possèdent une activité $\varepsilon 3$ ligase, pourraient participer au processus [35]. L'augmentation de la quantité de protéine NLRP3 lors du priming transcriptionnel, repose également en partie, sur la répression de l’£3 ligase SCF ${ }^{\text {FXLL2 }}$ (Skp-cullin-F box L2) responsable de l'ubiquitination de NLRP3 sur la lysine K689 qui conduit à sa dégradation par le protéasome. L'expression de l’£3 ligase $\mathrm{SCF}^{\mathrm{FBO} 03}$ est induite lors du priming transcriptionnel. Elle réprime $\mathrm{SCF}^{\mathrm{FXXL2}}$ par ubiquitination et dégradation [36]. À noter qu'au cours du priming transcriptionnel, la pro-IL-1 $\beta$ est également ubiquitinée sur la lysine K133 par des enzymes qui restent cependant à identifier. Cette ubiquitination semble favoriser le clivage de la cytokine par la caspase-1 [37]. Aucune modification similaire n'a été rapportée à notre connaissance pour les autres substrats de l'inflammasome, comme la proIL-18 ou la Gasdermine-D, et l'ubiquitination ne semble pas être un mécanisme commun d'adressage des substrats vers l'inflammasome.

L'étape d'activation de l'inflammasome repose aussi sur des modifications post-traductionnelles de NLRP3. L'assemblage de l'inflammasome nécessite en effet la déubiquitination de NLRP3 par la déubiquitinase BRCC3 (BRCA1/BRCA2-containing complex subunit 3) en aval de tous les activateurs qui ont été testés [38]. Cette déubiquitination semble donc constituer un élément clé de l'activation de l'inflammasome. Contrairement à celle impliquée dans le priming, elle est indépendante des ROS mitochondriales. Elle repose donc sur des voies de signalisation différentes [20]. Les domaines NACHT et LRR peuvent être ubiquitinés, et NLRP3 présente un mélange de chaînes d'ubiquitines de type $K 48$ et K63 [38]. BRCC3, spécifique des chaînes K63, cible le 


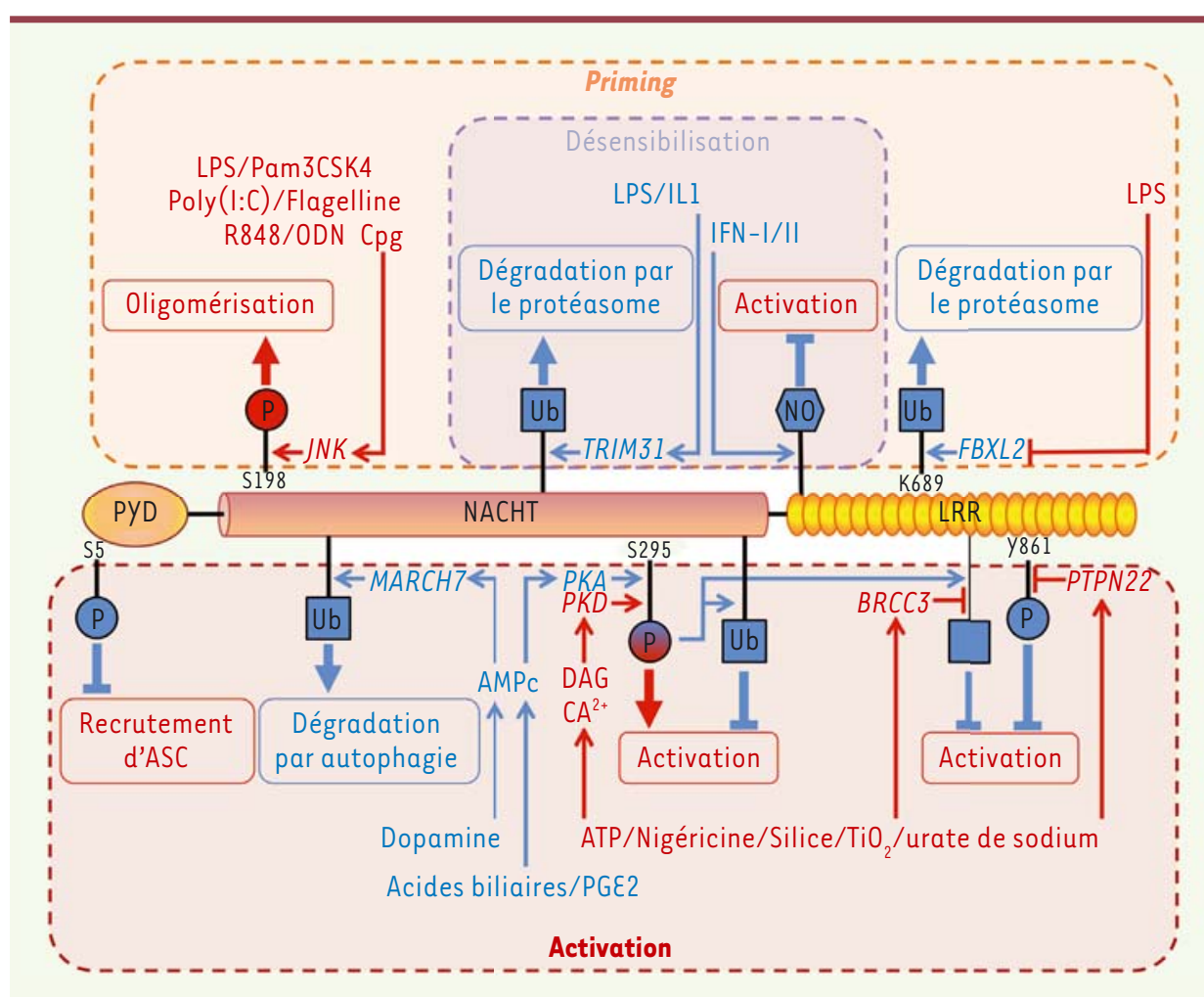

Figure 2. Modifications post-traductionnelles de NLRP3 impliquées dans la régulation de l'inflammasome. NLRP3 est composé des domaines PYD, NACHT et LRR modifiés par ubiquitination (Ub), phosphorylation $(P)$ et nitrosylation (NO) au cours des phases de priming, d'activation et de désensibilisation de NLRP3. Les enzymes responsables de ces modifications sont représentées en italique. Les stimulus et enzymes activateurs sont indiqués en rouge. Les stimulus, seconds messagers, enzymes et modifications inhibiteurs sont indiqués en bleu. NLRP3: NACHT, $\angle R R$ and PYD domains-containing protein 3 ; PYD : pyrin domain; LRR: leucin-rich repeats; LPS :lipopolysaccharide; Pam3CSK4, synthetic triacylated lipoprotein $\mathrm{C}_{81} \mathrm{H}_{156} \mathrm{~N}_{10} \mathrm{O}_{13} \mathrm{~S}$; Poly (I:C) : polyinosinic-polycytidylic acid;

R848 : resiquimod ; ODN CPG : oligodéoxynucléotide CPG ; ILI : interleukine-1 ; IFN-I : interféron de type 1 ; IFN-II : interféron de type 2 ; PGE2 : prostaglandine $\varepsilon 2$; ATP : adenosine triphosphate ; $\mathrm{TiO}_{2}$ : dioxyde de titane ; AMPc : cyclic adenosine monophosphate ; DAG : diacyl glycerol ; JNK : c-Jun N-terminal kinase ; TRIM31 : tripartite motif containing 31 ; FBXL2 : Skp-cullin-F box L2 ; MARCH7 : membrane-associated ring-CH-type finger 7 ; PKD : protein kinase D; PKA : protein kinase A; BRCC3 : BRCAl/BRCA2-containing complex subunit 3 ; PTPN22 : protein tyrosine phosphatase non-receptor type 22.

domaine LRR de NLRP3. Cependant, ni les lysines ubiquitinées, ni les conséquences biochimiques et structurales de cette ubiquitination ne sont connues. Dans des conditions d'inhibition de BRCC3, I'ubiquitination de NLRP3 empêche l'assemblage de l'inflammasome; elle n'est cependant pas associée à sa dégradation. Des événements de déphosphorylation sont également impliqués dans l'activation de NLRP3. Ainsi, la phosphatase PTPN22 (protein tyrosine phosphatase non-receptor type 22), qui a été associée à plusieurs pathologies inflammatoires, participe à l'activation de NLRP3, en aval d'activateurs de nature variée. Elle constitue un autre élément générique du processus. PTPN22 cible la tyrosine Y861 dans le domaine LRR de NLRP3. Son recrutement nécessite ASC. II est donc probablement consécutif à l'association préalable de NLRP3 et d'ASC [39]. Des substitutions (y861H et Y861C) au sein du domaine LRR ont été identifiées chez certains patients présentant un CAPS, suggérant l'importance de ces résidus dans le contrôle de l'activation de NLRP3 [40]. L'assemblage de l'inflammasome nécessite également la déphosphorylation de la sérine $\mathrm{S} 5$ du domaine PyD, qui permet le recrutement d'ASC. Cette déphosphorylation pourrait être réalisée par PP2A (protein phosphatase $2 A$ ) mais savoir si elle se produit lors du priming ou lors de l'activation n'est pas défini [41]. La phosphorylation de NLRP3 pourrait également réguler l'étape d'activation. Plusieurs kinases interagissent en effet avec NLRP3 et participent à son activation, mais leurs rôles dans sa phosphorylation ne sont pas établis. En particulier NEK7 (NIMA-related protein kinase 7), qui se lie à NLRP3, est nécessaire à l'assemblage de l'inflammasome auquel elle participe, mais son activité kinase n'est pas essentielle [42]. Très récemment, il a été montré que la phosphorylation de la sérine S295 de NLRP3 par les PKD (protein kinase D) 1-3, activées en aval de la PLC et du DAG (diacylglycérol) serait nécessaire à l'assemblage de l'inflammasome [43]. Les mutants S295A et S295E, qui miment respectivement les états déphosphorylé et phosphorylé de cette sérine, sont tous deux inactifs. Ce résultat suggère qu'un cycle de déphosphorylation/phosphorylation se produirait sur ce résidu. L'analyse de la localisation des mutants suggère que la déphosphorylation de la sérine S295 permettrait le recrutement initial de NLRP3 dans les MAM (mitochondria-associated $E R$ membranes). Sa phosphorylation par les PKDl-3 contrôlerait ensuite sa relocalisation, des MAM vers le speck cytosolique. Ce modèle est cohérent avec des études précédentes qui montrent l'inhibition de l'inflammasome par la voie dépendant de I'AMPc, qui est activée par les acides 
biliaires, via le récepteur TGR5 (Takeda G-protein-coupled receptor 5), et par l'eicosanoïde PGE2 (prostaglandin $\varepsilon 2$ ), via son récepteur EP4 ( $\varepsilon$ prostanoïd 4). En effet, cette inhibition repose sur la phosphorylation par la PKA de la sérine S295, ce qui conduit à l'ubiquitination de NLRP3 sans qu'il ne soit dégradé $[44,45]$. Le mutant S295A avait été décrit pour être actif, mais ce résultat, contradictoire, pourrait s'expliquer par le système de surexpression utilisé par les auteurs [45]. De nombreuses mutations identifiées chez les patients présentant un CAPS intéressent des sites à proximité de la sérine 295 et rendent NLRP3 insensible à l'inhibition par l'AMPc. Elles bloquent (au moins pour l'une d'entre elles) la phosphorylation par la PKA, suggérant un rôle important de ce point de régulation [45]. La répression par l'AMPc semble reposer sur plusieurs mécanismes. Dans le contexte de l'inhibition de NLRP3 par la dopamine via son récepteur DRD1 (dopamin receptor D1), I'AMPc se lie à NLRP3 et induit l'ubiquitination des domaines NACHT et LRR par l'£3 ligase MARCH7 (membrane-associated ring-CH-type finger 7). Ces ubiquitinations conduisent à la dégradation de NLRP3 par autophagie [46].

L'assemblage de l'inflammasome pourrait être également modulé par des modifications touchant d'autres éléments qui le constituent. Notamment, la caspase-1 peut être ubiquitinée par clAP2 (cellular inhibitor of apoptosis 2) dont l'expression favorise l'activation de NLRP3. ASC est également ubiquitiné par LUBAC (linear ubiquitin chain assembly complex) et est phosphorylé sur plusieurs sites au cours du priming et/ou de l'activation. L'impact de ces différentes modifications sur l'activation de l'inflammasome demeure cependant incertain [47-49]. L'ubiquitination d'ASC par des chaînes d'ubiquitines de type $K 63$ pourrait également être impliquée dans la dégradation de l'inflammasome par autophagie et l'arrêt de la réponse de la cellule à la fin du processus d'activation [50].

La désensibilisation de NLRP3, après un long engagement de certains des récepteurs de priming, repose sur des modifications qui l'altèrent. Ainsi, l'£3 ligase TRIM31 (tripartite motif containing 31), induite par le lipopolysaccharide bactérien (LPS) et l'IL-1, induit I'ubiquitination de NLRP3 avec des chaînes K48, ce qui conduit à sa dégradation par le protéasome [51]. La sécrétion autocrine/paracrine d'IFN-I conduit à la production de NO, en activant l'iNOS (inducible nitric oxyde synthase) responsable de la S-nitrosylation de NLRP3 au niveau de son domaine LRR riche en cystéines, qui inhibe l'assemblage de l'inflammasome [22]. L'IFN- $\gamma$ (un interféron de type II) inhibe aussi NLRP3, par le même mécanisme [52].

\section{Conclusion}

La dérégulation du récepteur NLRP3 est impliquée dans de nombreuses pathologies à composante inflammatoire. L'inhibition de l'inflammasome NLRP3 porte ainsi d'importants espoirs thérapeutiques. Les voies de signalisation qui contrôlent son assemblage restent cependant insuffisamment comprises. Récemment, les modifications post-traductionnelles de NLRP3 se sont néanmoins révélées être des points clés de sa régulation. L'identification de la nature de ces modifications, des résidus qui sont concernés, et, surtout, des complexes enzymatiques responsables permettront, dans l'avenir, de mieux comprendre les mécanismes d'assemblage de l'inflammasome et, nous l'espérons, d'identifier de nouvelles cibles thérapeutiques. $\diamond$

\section{SUMMARY}

\section{Regulation of the NLRP3 inflammasome}

The innate immunity constitutes an efficient barrier by rapidly detecting pathogens and tissue damages through pattern recognition receptors including NLRP3. Moreover, inappropriate NLRP3 activation causes deleterious inflammation and contributes to various conditions including atherosclerosis, diabetes, gout and Alzheimer's diseases. NLRP3 assembles a multimeric inflammasome complex serving as an activation platform for caspase- 1 that controls processing and release of cytosolic inflammatory factors and cytokines including IL-1 $\beta$. Inflammasome assembly is tightly controlled and requires coordinated NLRP3 priming, through cytokine or other pattern recognition receptors, followed by activation by cellular stress. Here, we describe recent advances in the understanding of the signalling pathways supporting the priming and activation of NLRP3, with a special focus on the key role of post-translational modifications of NLRP3, including phosphorylation and ubiquitination, in inflammasome regulation. $\diamond$

\section{REMERCIEMENTS}

Ces travaux ont été financés en partie par l'Agence Nationale pour la Recherche (ANR-13-JSV3-0002-01) et l'European Research Council (ERC-2013-CoG_616986) (B.P). Les auteurs remercient Amaury Rey pour la relecture critique du manuscrit.

\section{LIENS D'INTÉRÊT}

Les auteurs déclarent n'avoir aucun lien d'intérêt concernant les données publiées dans cet article.

\section{RÉFÉRENCES}

1. Jamilloux Y, Henry T. Les inflammasomes : plates-formes de l'immunité innée. Med Sci (Paris) $2013 ; 29$ : 975-84.

2. Kayagaki N, Stowe IB, Lee BL, et al. Caspase-11 cleaves gasdermin D for non-canonical inflammasome signalling. Nature 2015; 526 : 666-71.

3. Cabon L, Martinez-Torres AC, Susin SA. La mort cellulaire programmée ne manque pas de vocabulaire. Med Sci (Paris) $2013 ; 29$ : 1117-24.

4. Lu A, Magupalli VG, Ruan J, et al. Unified polymerization mechanism for the assembly of ASC-dependent inflammasomes. Cell 2014 ; 156 : 1193-206.

5. Shinkai K, McCalmont TH, Leslie KS. Cryopyrin-associated periodic syndromes and autoinflammation. Clin Exp Dermatol 2008 ; 33 : 1-9.

6. Martinon F, Pétrilli V, Mayor A, et al. Gout-associated uric acid crystals activate the NALP3 inflammasome. Nature 2006 ; $440: 237-41$.

7. Vandanmagsar B, Youm $Y H$, Ravussin A, et al. The NALP3/NLRP3 inflammasome instigates obesity-induced autoinflammation and insulin resistance. Nat Med $2011 ; 17: 179-88$.

8. Youm Y-H, Grant RW, McCabe LR, et al. Canonical Nlrp3 inflammasome links systemic low grade inflammation to functional decline in aging. Cell Metab $2013 ; 18: 519-32$.

9. Heneka MT, Kummer MP, Latz $\varepsilon$. Innate immune activation in neurodegenerative disease. Nat Rev Immunol 2014 ; 14 : 463-77.

10. Franchi L, Muñoz-Planillo R, Núñez G. Sensing and reacting to microbes via the inflammasomes. Nat Immunol $2012 ; 13: 325-32$. 


\section{RéFÉRENCES}

11. Dostert C, Pétrilli V. Amiante et inflammation, rôle de l'inflammasome. Med Sci (Paris) 2008 ; 24 916-8.

12. Elliott $\varepsilon 1$, Sutterwala FS. Initiation and perpetuation of NLRP3 inflammasome activation and assembly. Immunol Rev. $2015 ; 265: 35-52$

13. Gaidt MM, Ebert TS, Chauhan $D$, et al. Human monocytes engage an alternative inflammasome pathway. Immunity $2016 ; 44: 833-46$

14. Jamilloux Y, Lefeuvre L, Magnotti F, et al. Familial Mediterranean fever mutations are hypermorphic mutations that specifically decrease the activation threshold of the Pyrin inflammasome. Rheumatology 2017 ; doi: 10.1093/rheumatology/kex373.

15. Bauernfeind FG, Horvath G, Stutz A, et al. Cutting edge: NF-kappaB activating pattern recognition and cytokine receptors license NLRP3 inflammasome activation by regulating NLRP3 expression. Immunol 2009; $183:$ 787-91.

16. Franchi L, Eigenbrod T, Núñez G. Cutting edge: TNF-alpha mediates sensitization to ATP and silica via the NLRP3 inflammasome in the absence of microbial stimulation. J Immunol $2009 ; 183: 792$

17. Gurung P, Anand PK, Malireddi RKS, et al. FADD and caspase-8 mediate priming and activation of the canonical and noncanonical Nlrp3 inflammasomes. J Immunol 2014 ; 192 : 1835-46.

18. Zhang $X$, Fan C, Zhang $H$, et al. MLKL and FADD are critical for suppressing progressive lymphoproliferative disease and activating the NLRP3 inflammasome. Cell Rep 2016 ; 16 : 324759

19. Fernandes-Alnemri T, Kang S, Anderson C, et al. Cutting edge: TLR signaling licenses IRAKI for rapid activation of the NLRP3 inflammasome. J Immunol 2013 ; 191 : 3995-9.

20. Juliana $C$, Fernandes-Alnemri T, Kang $S$, et al. Non-transcriptional priming and deubiquitination regulate NLRP3 inflammasome activation. J Biol Chem 2012 ; 287 : 36617-22.

21. Kang S, Fernandes-Alnemri T, Rogers $C$, et al. Caspase- 8 scaffolding function and MLKL regulate NLRP3 inflammasome activation downstream of TLR3. Nat Commun 2015; $6: 7515$.

22. Hernandez-Cuellar $\varepsilon$, Tsuchiya $K$, Hara H, et al. Cutting edge: nitric oxide inhibits the NLRP3 inflammasome. J Immunol $2012 ; 189$ : 5113-7.

23. Rühl S, Broz P. Caspase-11 activates a canonical NLRP3 inflammasome by promoting $\mathrm{K}^{+}$efflux. Eur J Immunol $2015 ; 45: 2927-36$.

24. Solle M, Labasi J, Perregaux DG, et al. Altered cytokine production in mice lacking P2X(7) receptors. J Biol Chem $2001 ; 276$ : 125-32.

25. Hornung V, Bauernfeind F, Halle A, et al. Silica crystals and aluminum salts mediate NALP-3 inflammasome activation via phagosomal destabilization. Nat Immunol $2008 ; 9: 847-56$.

26. Franklin BS, Bossaller L, De Nardo D, et al. The adaptor ASC has extracellular and prionoid activities that propagate inflammation. Nat Immunol $2014 ; 15: 727-37$.

27. Baroja-Mazo A, Martín-Sánchez F, Gomez Al, et al. The NLRP3 inflammasome is released as a particulate danger signal that amplifies the inflammatory response. Nat Immunol 2014 ; 15 : 738 48.

28. Zhou R, Yazdi AS, Menu P, et al. A role for mitochondria in NLRP3 inflammasome activation. Nature $2011 ; 469: 221-5$.

29. Lee GS, Subramanian N, Kim Al, et al. The calcium-sensing receptor regulates the NLRP3 inflammasome through $\mathrm{Ca}^{2+}$ and cAMP. Nature $2012 ; 492: 123-7$.

30. Muñoz-Planillo R, Kuffa P, Martínez-Colón $G$, et al. $K^{+}$efflux is the common trigger of NLRP3 inflammasome activation by bacterial toxins and particulate matter. Immunity $2013 ; 38: 1142$ 53.

31. Iyer SS, He Q, Janczy JR, et al. Mitochondrial cardiolipin is required for Nlrp3 inflammasome activation. Immunity $2013 ; 39: 311-23$

32. Nakahira K, Haspel JA, Rathinam VA, et al. Autophagy proteins regulate innate immune response by inhibiting NALP3 inflammasome-mediated mitochondrial DNA release. Nat Immunol 2011 ; 12 222-30.

33. Song N, Liu ZS, Xue W, et al. NLRP3 Phosphorylation is an essential priming event for inflammasome activation. Mol Cell $2017 ; 68: 185-7$.

34. Saito M, Fujisawa A, Nishikomori R, et al. Somatic mosaicism of CIAS1 in a patient with chronic infantile neurologic, cutaneous, articular syndrome. Arthritis Rheum $2005 ; 52: 3579-85$.
35. Mayor A, Martinon F, De Smedt T, et al. A crucial function of SGTl and HSP9O in inflammasome activity links mammalian and plant innate immune responses. Nat Immunol $2007 ; 8: 497-503$.

36. Han S, Lear TB, Jerome JA, et al. Lipopolysaccharide primes the NALP3 inflammasome by inhibiting its ubiquitination and degradation mediated by the SCFFBXL2 \&3 ligase. J Biol Chem 2015 ; 290 : 18124-33.

37. Duong BH, Onizawa $M$, Oses-Prieto JA, et al. A20 restricts ubiquitination of pro-interleukin- $1 \beta$ protein complexes and suppresses NLRP3 inflammasome activity. Immunity $2015 ; 42: 55-67$.

38. Py BF, Kim M-S, Vakifahmetoglu-Norberg H, et al. Deubiquitination of NLRP3 by BRCC3 critically regulates inflammasome activity. Mol Cell $2013 ; 49$ : $331-8$

39. Spalinger MR, Kasper S, Gottier C, et al. NLRP3 tyrosine phosphorylation is controlled by protein tyrosine phosphatase PTPN22. J Clin Invest 2016; 126 : $1783-1800$

40. Frenkel J, Kempen MJA van, Kuis W, et al. Variant chronic infantile neurologic, cutaneous, articular syndrome due to a mutation within the leucine-rich repeat domain of CIAS1. Arthritis Rheum 2004 ; 50 : 2719-20.

41. Stutz A, Kolbe CC, Stahl R, et al. NLRP3 inflammasome assembly is regulated by phosphorylation of the pyrin domain. J Exp Med 2017 ; 214 : 1725-36.

42. He Y, Zeng MY, Yang D, et al. Nek7 is an essential mediator of NLRP3 activation downstream of potassium efflux. Nature $2016 ; 530: 354-7$.

43. Zhang Z, Meszaros G, He WT, et al. Protein kinase D at the Golgi controls NLRP3 inflammasome activation. J Exp Med 2017 ; 214 : 2671-93.

44. Guo C, Xie S, Chi Z, et al. Bile acids control inflammation and metabolic disorder through inhibition of NLRP3 inflammasome. Immunity 2016; 45 : 802-16.

45. Mortimer L, Moreau F, MacDonald JA, et al. NLRP3 inflammasome inhibition is disrupted in a group of auto-inflammatory disease CAPS mutations. Nat Immunol 2016; 17 : 1176-86.

46. Yan $Y$, Jiang W, Liu L, et al. Dopamine controls systemic inflammation through inhibition of NLRP3 inflammasome. Cell 2015; 160:62-73.

47. Hara H, Tsuchiya K, Kawamura I, et al. Phosphorylation of ASC acts as a molecular switch controlling the formation of speck-like aggregates and inflammasome activity. Nat Immunol 2013; 14 : 1247-55.

48. Labbé K, McIntire CR, Doiron K, et al. Cellular inhibitors of apoptosis proteins CIAPI and CIAP2 are required for efficient caspase- 1 activation by the inflammasome. Immunity $2011 ; 35: 897-907$.

49. Rodgers MA, Bowman JW, Fujita $\mathrm{H}$, et al. The linear ubiquitin assembly complex (LUBAC) is essential for NLRP3 inflammasome activation. J Exp Med $2014 ; 211: 1333-47$

50. Shi CS, Shenderov K, Huang NN, et al. Activation of autophagy by inflammatory signals limits IL- $1 \beta$ production by targeting ubiquitinated inflammasomes for destruction. Nat Immunol 2012; 13 : 255-63.

51. Song H, Liu B, Huai W, et al. The $\varepsilon 3$ ubiquitin ligase TRIM31 attenuates NLRP3 inflammasome activation by promoting proteasomal degradation of NLRP3. Nat Commun $2016 ; 7: 13727$.

52. Mishra BB, Rathinam VAK, Martens GW, et al. Nitric oxide controls the immunopathology of tuberculosis by inhibiting NLRP3 inflammasomedependent processing of IL-1 $\beta$. Nat Immunol $2013 ; 14$ : 52-60.

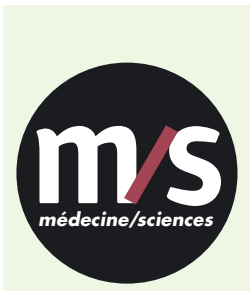

Tarifs d'abonnement $m / s-2018$

$>$ Grâce à $m / s$, vivez en direct les progrès des sciences biologiques et médicales

Abonnez-vous à médecine/sciences

Bulletin d'abonnement page 98 dans ce numéro de $\mathrm{m} / \mathrm{s}$ 